\title{
Open Clusters: Open Windows on Stellar Dynamics
}

\author{
Robert D. Mathieu ${ }^{1}$ \\ ${ }^{1}$ Department of Astronomy, University of Wisconsin - Madison, WI, 53706, USA \\ email: mathieu@astro.wisc.edu
}

\begin{abstract}
The extensive stellar radial-velocity surveys of the WIYN Open Cluster Study now allow comprehensive studies of the solar-type hard-binary populations in open clusters as a function of age. We first describe an empirical "initial" hard-binary population as derived from the young open cluster NGC 2168 (M35). Given the limited analyses so far, the cluster binary population is indistinguishable from that of the field. We then compare the hard-binary population in the old open cluster NGC 188 to the binary population in the sophisticated N-body simulations of the old cluster M67 by Hurley et al. The binary populations in the cluster and the simulation show significant differences in binary frequency and fraction of circularized binaries, while otherwise showing similar orbital eccentricity distributions. Since the simulations were designed to match the encounter products in M67, such as blue stragglers, the large reduction in binary fraction indicated by the empirical results likely will also require changes in the simulation physics producing blue stragglers and other anomalous stars arising from stellar dynamics. We present three case studies of stars in open clusters which very likely are products of dynamical encounters between binaries and either single stars or other binaries: the M67 blue straggler S1082, the M67 sub-subgiant S1113, and the horizontal branch star 6819-3002 in the intermediate-age open cluster NGC 6819. Finally, we remind the reader of recent empirical results on the rates of tidal interactions, using tidal circularization periods in open clusters. Every indication is that current theories underestimate the effectiveness of tidal circularization, a result that need to be incorporated into dynamical simulations of dense stellar systems.
\end{abstract}

Keywords. Stars: spectroscopic binaries, blue stragglers, evolution; Galaxy: open clusters; Physical data and processes: stellar dynamics

\section{Introduction}

It might be argued that open clusters are not an appropriate subject for a meeting on the dynamical evolution of dense stellar systems, since by most dynamical measures open clusters are not particularly dense. Nonetheless open clusters are highly accessible laboratories for many of the processes that take place in dense stellar systems. Furthermore, current numerical techniques permit full-scale dynamical simulations of open clusters, even including reasonable approximations of dissipative stellar interactions. As such, they are superb testbeds for the codes that will be used to model dense stellar systems in the near future.

The WIYN Open Cluster Study (WOCS; Mathieu 2000) has been intensively observing a set of select open clusters to obtain complete and accurate descriptions of their populations, spatial distributions, and internal kinematics. The contribution of the University of Wisconsin - Madison team has been primarily highly precise $\left(\sigma \sim 0.4 \mathrm{~km} \mathrm{~s}^{-1}\right)$ radial-velocity surveys providing multiple measurements for stars over time spans of a decade and longer. As cases in point, as of August 2007 the WOCS database includes 8572 radial-velocity measurements of 1092 stars in the field of the open cluster NGC 188, 7698 measurements of 1517 stars in the field of NGC 6819, and 7104 measurements of 
1597 stars in the field of NGC 2168 (M35). More than 100 velocity variables have been identified in each of these clusters, with most having spectroscopic orbit solutions.

Such data are now ready to speak to broad questions in stellar dynamics, stellar evolution, and most interestingly the interfaces between these two classical fields. Here I give a first glimpse at the imminent prospects.

\section{Hard Binary Populations in Open Clusters}

Two of the more advanced WOCS studies are those of the young cluster NGC 2168 (0.15 Gyr) and the very evolved cluster NGC 188 (7 Gyr). Here we demonstrate the current possibilities for detailed comparisons of clusters and simulations, in this case in the context of hard-binary populations.

\section{1. "Initial" Hard-Binary Population}

The initial conditions of most N-body simulations are not truly primordial, of course, in that the star- and cluster-formation events are rarely included. (Although it is exciting to see that simulations from cluster birth to death will be in our very near future (e.g., C. Clarke, this volume).) Rather, many simulations start from a configuration that includes a mass spectrum of main-sequence stars bound within a near-equilibrium cluster. A critical initial condition is the binary population, including descriptions of binary frequency, period distribution, orbital eccentricity distribution, secondary mass function, and the like. To date the initial binary population often has been characterized by equilibrium expectations or integrations of equilibrium expectations with field binary observations (e.g., Kroupa 1995). The WOCS study of the young open cluster NGC 2168 provides an empirical initial binary population. We summarize here several key findings - more details can be found in Braden et al. (this volume, and in preparation).

We take the field solar-type binary population of Duquennoy \& Mayor (1991; DM) as the null hypothesis against which we compare the NGC 2168 binary population. In Fig. 1 we compare the NGC 2168 period and eccentricity distributions with those of DM, scaled appropriately by total sample size. For periods of less than 100 days the field and cluster frequency distributions are indistinguishable. At greater than 100 days the cluster distribution does not continue to rise as does the field. This is likely an effect of incompleteness in binary orbit solutions; we should achieve completeness through periods of 1000 days within a year or two.

The M35 orbital eccentricity distribution shows a significant population of circular orbits, almost certainly the result of tidal circularization processes (see Section 4). For e $>0.1$, we compare the cluster eccentricity distribution with a Gaussian fit to the DM eccentricity distribution (in the DM case, for binaries with periods between the tidal circularization cutoff period and 1000 days). Both show distributions that decrease at low and high eccentricities. The DM distribution peaks at an eccentricity of 0.3 , somewhat shorter than the peak in the cluster distribution. Whether this difference is meaningful remains to be seen; certainly to zeroth order it is the similarity of the cluster and field eccentricity distributions that is most significant.

Finally in Fig. 2 we show the radial spatial distribution of single and binary solar-type stars in NGC 2168, which are not evidently different. Mathieu (1983) and McNamara \& Sekiguchi (1986) found that the most massive stars in the cluster are mass segregated, but the degree of segregation lessens for masses of 1-1.5 $\mathrm{M}_{\odot}$. More detailed dynamical modeling is merited to see whether the similar radial distributions of the single and binary solar-type stars are in equilibrium with the more centrally concentrated distributions of the massive stars. 

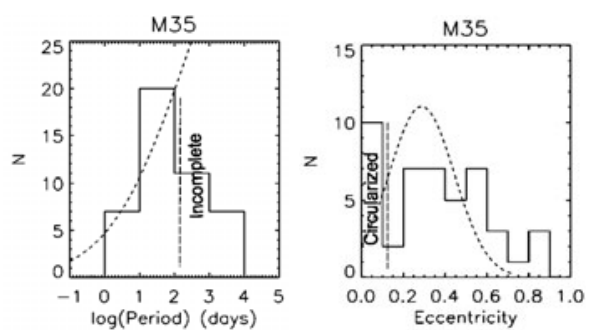

Figure 1. Left: The period distribution of spectroscopic binaries in the young open cluster NGC 2168. The dashed line represents the field period distribution of Duquennoy \& Mayor (1991), scaled by sample size. Right: The orbital eccentricity distribution of short-period binaries in NGC 2168. Again, the dashed line represents the field eccentricity distribution of DM, scaled by sample size.

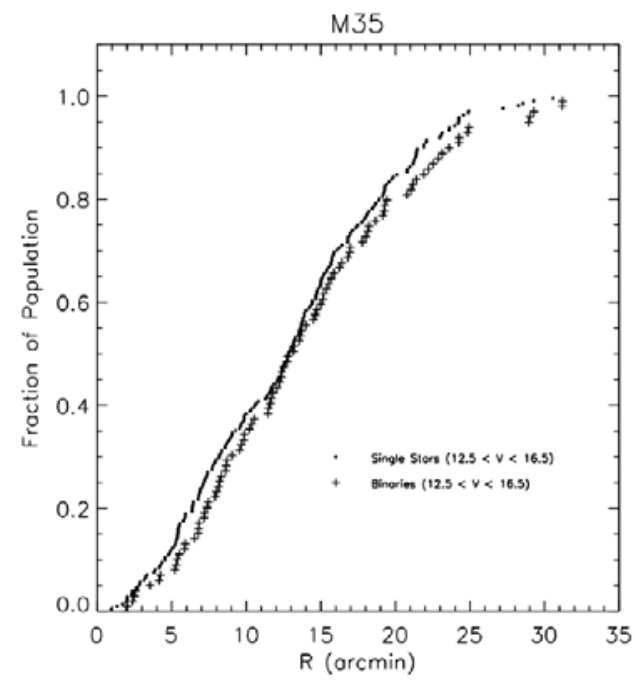

Figure 2. The cumulative radial distributions of solar-type single stars and binary stars in the young open cluster NGC 2168. The two distributions are indistinguishable.

\subsection{Evolved Hard-Binary Populations}

The most detailed N-body simulation of an open cluster is that of Hurley et al. (2005), who sought to create a cluster of age 4.5 Gyr that could be considered as a match (statistically speaking) to the open cluster M67. Importantly, Hurley et al. had little a priori knowledge of the current M67 binary population; their primary interfaces between their models and M67 were the current color-magnitude diagram, mass function, and population of blue stragglers. As such, the current binary population of M67 represents a powerful post facto test of the dynamical models.

We are in the process of defining the current M67 binary population. Here in its place we use the binary population of NGC 188, an older open cluster with an age of 7 Gyr. We summarize here several key findings - more details can be found in Geller et al. (this volume, and 2008).

The period distributions of both NGC 188 and the M67 simulation are shown in Fig. 3. Here we have adapted the M67 simulation to match our observational constraints specifically, a mass range of greater than $\sim 0.9 \mathrm{M}_{\odot}$, a $15 \mathrm{pc}$ diameter region, and the inclusion only of binaries with periods less than $10^{4}$ days. Finally in Fig. 3 is shown the scaled DM distribution as a reference for comparison.

Clearly, the binary frequency of the M67 simulation is much higher than the actual binary population of NGC 188 (by a factor 6 at a period of 10 days). We suspect that this difference is primarily linked to the high initial binary frequency of the simulation rather than dynamical evolution. Importantly, this high binary frequency was needed to reproduce the M67 blue straggler population, so significantly lowering it to match the observed binary populations of either NGC 2168 or NGC 188 also will require changes to the physics of blue straggler creation.

The decrease in frequency for periods greater than 100 days of the binary population of the M67 simulation is the result of dynamical evolution. Again, the NGC 188 population may not be complete at periods longer than 100 days, so noting the similar drop in the NGC 188 distribution is likely premature. In addition to adding a decade of completeness 

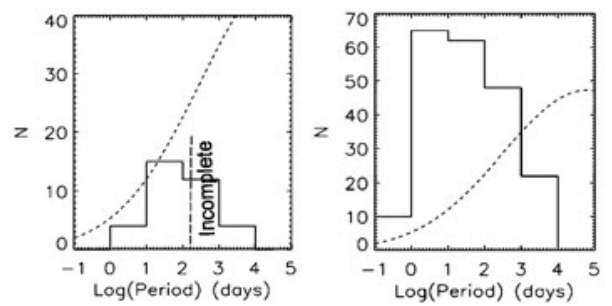

Figure 3. The period distribution of hard binaries in NGC 188 (left) and from simulated observations of an N-body model of M67 (Hurley et al. 2005). The dashed line represents the field period distribution of DM, scaled by sample size.
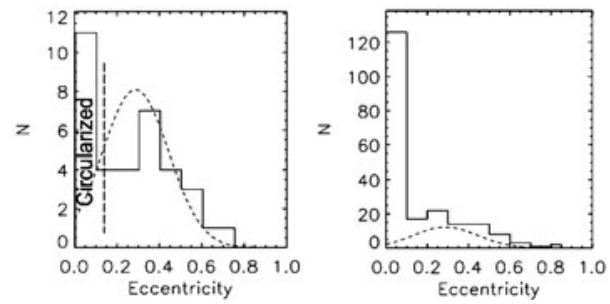

Figure 4. The orbital eccentricity distribution of hard binaries in NGC 188 (left) and simulated observations of an N-body model of M67 (Hurley et al. 2005). The dashed line represents the field eccentricity distribution of Duquennoy \& Mayor (1991), scaled by sample size.

to the NGC 188 period distribution, we hope to soon use photometric techniques to get a handle on the total binary frequency of NGC 188. This also will allow us to constrain the dynamical evolution of binaries in the cluster (Frinchaboy \& Nielsen, this volume.)

However, we can already examine the evolution of hard-binary frequencies as a function of cluster age. Specifically, we consider the frequency of solar-type binaries within 4 core radii whose radial-velocity measurements produce a standard deviation greater than $2 \mathrm{~km} \mathrm{~s}^{-1}$. The frequency is $33 \% \pm 3 \%$ in NGC 2168 (0.15 Gyr), 37\% $\pm 3 \%$ in NGC 6819 (2.4 Gyr), and $35 \% \pm 3 \%$ in NGC 188 ( 7 Gyr). Thus we find remarkably little evolution of the hard binary frequency over 7 Gyr. (The frequency is $58 \% \pm 4 \%$ in the M67 simulation, much higher as already noted.)

In Fig. 4 we show the orbital eccentricity distributions for both NGC 188 and for simulated observations of the M67 model. Clearly there is a striking excess of circularized binaries in the simulation. The frequency of circularized binaries in NGC 188 compares well with those in other old open clusters, including M67 (Mathieu et al. 2004). Curiously, current tidal circularization theories underestimate the tidal circularization cutoff periods in these older clusters. Thus the presence of so many circularized binaries in the M67 simulation is somewhat of a surprise that still needs to be understood.

For non-zero eccentricities, the agreement of the two eccentricity distributions is reasonably good. Indeed, both NGC 188 and the M67 simulation show eccentricity distributions much like that found by DM for field solar-type binaries. This is notable in that the cluster binaries have been immersed in a denser stellar environment than the field binaries, with consequent dynamical encounters and evolution.

Finally, we note that the binaries and the blue stragglers of both NGC 188 and the M67 simulation are both centrally concentrated with respect to the single stars (or, more strictly, the stars not showing measurable velocity variation; Geller et al.). Curiously, though, the giant stars in the M67 simulation are centrally concentrated while the giants in both NGC 188 and M67 are not (Geller et al., this volume; Mathieu 1983, Tinsley \& King 1976).

\section{An Array of Anomalous Stars - The Interface of Stellar Dynamics and Stellar Astrophysics}

I suspect that in every astronomy survey course around the world a figure is shown to the students that overlays main sequences and giant branches from a set of clusters of varying ages, thereby demonstrating stellar evolution. Often, the cluster sequences are 

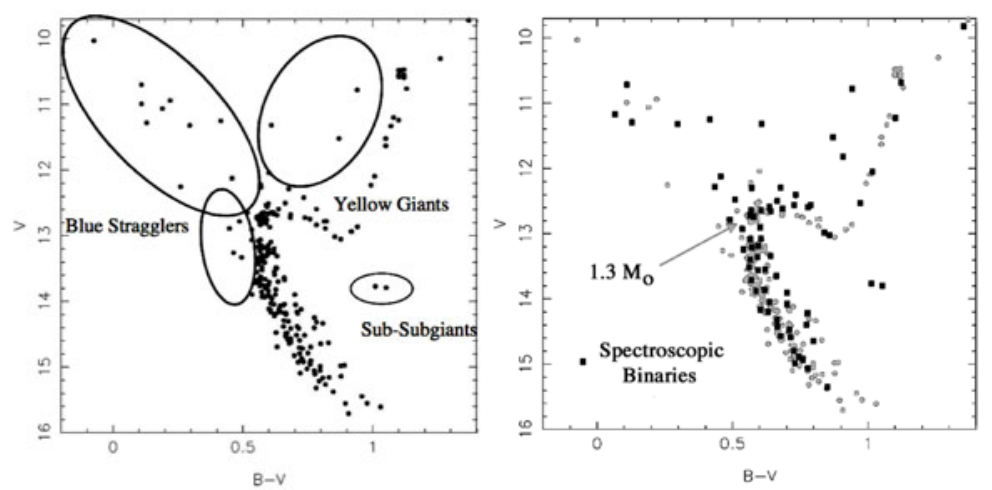

Figure 5. Left: A color-magnitude diagram of M67. All stars shown are three-dimensional kinematic cluster members. Right: The same figure with all known spectroscopic binaries shown as black square symbols.

simplified to show only the traces of the main sequence, the subgiants if appropriate, and the giants. While perhaps pedagogically useful, this approach can mislead our students into thinking that stellar evolution theory is essentially complete and, while impressive in its success, no longer at the forefront as a field of research.

In reality, the color-magnitude diagram of M67 looks like Fig. 5a. A substantial fraction of the cluster members do not lie on the classical single-star isochrone. Some of the members are not on the single-star locus simply because they are the combined light of two or more stars in multiple systems. But many of the stars are not so easily explained, as labeled in the figure. The blue stragglers of course have been known (if not understood) for a half-century. Also indicated in the figure is the possibility (indeed, the likelihood) that "blue stragglers" also exist below a cluster turnoff, either to the blue of or embedded within the main sequence. In addition to the blue stragglers there are "yellow giants" (e.g., Mathieu \& Latham 1986) and the recently discovered sub-subgiants (Mathieu et al. 2003).

Another important way to look at a cluster color-magnitude diagram is shown in Fig. 5b . Here we have shown all of the currently known spectroscopic binaries in M67. The figure shows graphically the fact that at least one third, and presumably a higher fraction, of the stars in a cluster color-magnitude diagram are binaries. As such, they have a much higher cross-section for dynamical encounters with other single stars and binaries, and a consequently higher chance of taking an evolutionary path different from isolated single stars. This interface of stellar dynamics and stellar evolution represent the exciting forefronts of both classical fields. Some examples...

\subsection{The Blue Straggler S1082}

In 1992, Goranskij et al. suggested that the star S1082 in M67 was an eclipsing binary. This discovery went largely unnoticed for a decade. Then both van den Berg et al. (2001) and Sandquist et al. (2003) studied the system extensively. Fig. 6 shows the outcome of the Sandquist et al. analysis of the system. The system is likely a hierarchical triple. The eclipsing binary has a period of 1.07 days, while the tertiary has a period of roughly 1000 days. Interestingly, both the tertiary and the primary of the eclipsing binary are blue stragglers. The secondary of the eclipsing binary lies on the main sequence, within substantial error bars. 


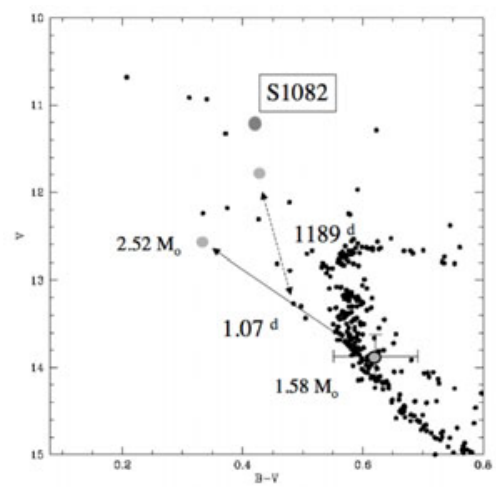

Figure 6. Location of the three spectroscopic components of the eclipsing blue straggler S1082 in the M67 color-magnitude diagram. (Adapted from Sandquist et al. 2003.)

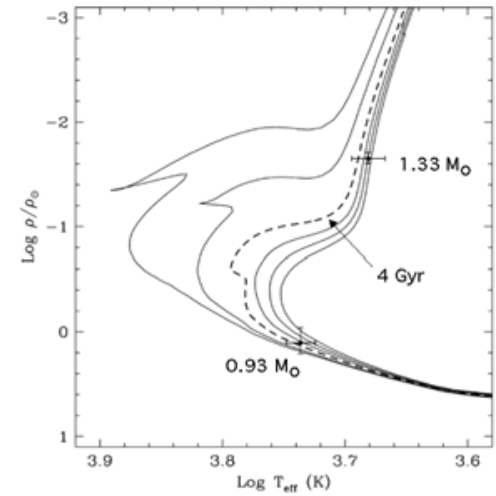

Figure 7. A set of isochrones in the log $\rho-\mathrm{T}_{\text {eff }}$ diagram. Also shown are the locations of the primary and secondary stars of the sub-subgiant binary S1113. The lower density datum represents the primary star, the other datum is the secondary star. The curves in the figure are solar-metallicity Yale isochrones (Kim et al. 2002) for ages 1, $2,4,6,8$, and 10 Gyr. The 4 Gyr isochrone, comparable with the age of M67, is shown as a dashed line. (Taken from Mathieu et al. 2003.)

With the addition of the stellar masses, the system becomes truly fascinating. The mass of the blue straggler primary of the eclipsing binary is $2.52 \mathrm{M}_{\odot}$, encouragingly greater than the cluster turnoff mass of $1.3 \mathrm{M}_{\odot}$. But most remarkably, the mass of the secondary is $1.58 \mathrm{M}_{\odot}-$ even though it lies on the main-sequence below the turnoff! Apparently we are not dealing with a simple single star in equilibrium.

At this point discussing an evolutionary scenario is more for the pleasure of speculation than for secure understanding. Remembering that the turnoff mass of M67 is $1.3 \mathrm{M}_{\odot}$, the presence of $4 \mathrm{M}_{\odot}$ in the eclipsing binary suggests an origin involving at least three stars. Including the blue straggler tertiary, van den Berg et al. (2001) suggested that the system formed from dynamical interactions of five stars or more, likely in multiple binary-binary encounters. In this scenario, during an encounter a resonant interaction of three stars leads two stars to collide and merge directly to the blue straggler primary of the eclipsing binary, with the third star becoming the current secondary. A fourth star becomes the widely separated current tertiary. That this star is also a blue straggler is challenging. Was it already such in one of the encountering binaries? What is the likelihood of this relative to a blue straggler lifetime? Or was it too formed from a collision during the formation of the current system?

From the point of view of stellar evolution, the most intriguing result may be the secondary star, lying on the main sequence with a mass substantially larger than the turnoff mass. Equally notable, the radius of the secondary is slightly larger than the radius of the more massive primary star. Both findings indicate that the secondary may not be in a thermal equilibrium state. This would require a rather recent formation, also suggested by the asynchronous rotation of at least the secondary despite the very short-period orbit.

Ultimately for this meeting the significance of S1082 is that it is one of the most convincing cases in support of dynamical encounters, and indeed stellar collisions, occurring in stellar systems. 


\subsection{The Sub-Subgiant S1113}

The M67 stars S1063 and S1113 have attracted attention for their location in the cluster color-magnitude diagram roughly 1 mag below the subgiant branch. Both stars are threedimensional kinematic members of the cluster. Mathieu et al. (2003) gave them the name of "sub-subgiants", while Albrow et al. (2001) call similarly located stars in the globular cluster 47 Tuc "red stragglers".

Both M67 sub-subgiants are spectroscopic binary stars; here we present S1113 in some detail. S1113 is a double-lined spectroscopic binary with a period of 2.8 days, a circular orbit, and a mass ratio of 0.7 . The system is photometrically variable, also with a period of 2.8 days. Presuming a spot origin for the variability, the identity of the orbital and photometric periods indicates synchronous rotation, as expected for such a short-period orbit.

Presuming synchronous rotation, the mean density of each star can be obtained directly from observables $\left(\mathrm{P}, \mathrm{v} \sin i\right.$, and $\left.\mathrm{M} \sin ^{3} i\right)$. In Fig. 7 we place both stars in a $\log \rho-$ $\log \mathrm{T}_{\text {eff }}$ diagram. Interestingly, both stars can be fit to a $4 \mathrm{Gyr}$ isochrone, with the secondary a $0.9 \mathrm{M}_{\odot}$ main-sequence star and the primary a $1.3 \mathrm{M}_{\odot}$ giant. However, such an interpretation of the system requires a luminosity ratio of 9 , while the measured luminosity ratio is only 3 . If the secondary is in fact a main-sequence star, then the primary star is underluminous - hence its location below the subgiant branch of the cluster. This low luminosity indicates a primary radius that is currently well within the associated Roche lobe.

As yet the case of S1113 awaits a satisfactory evolutionary scenario. Mathieu et al. (2003) conjecture that its evolutionary history includes mass transfer episodes, mergers, and/or dynamical stellar exchanges. To this might be added close encounters that may have stripped the primary envelope.

\subsection{The Horizontal Branch Star 6819-3002}

At first glance, star 3002 (WOCS identification number) in the red clump (i.e., the horizontal branch) of the intermediate-age open cluster NGC 6819 is not evidently noteworthy. It has a period of 17.7 days and a circular orbit; the latter is not a surprise given a giant primary at such an orbital period. Presuming that the primary has the turnoff mass of $1.6 \mathrm{M}_{\odot}$, the current separation is about $0.2 \mathrm{AU}$, so that at present the primary star fits comfortably within its Roche lobe.

However, given its present position in the red clump, the primary star would have already evolved up to the tip of the giant branch. Thus in the past the star would have had a radius approaching $50 \mathrm{R}_{\odot}$ (using the Yale isochrones), and far exceeded its Roche lobe if it were in the current binary. Presumably mass transfer would have precluded the star becoming a normal cluster horizontal branch star.

Thus we suggest that this system may be the result of a dynamical encounter, and specifically we hypothesize that the current horizontal branch primary star was exchanged into the system during a single star - binary encounter.

Gosnell et al. (2008) tested this hypothesis using the FEWBODY code of Fregeau et al. (2004) to run nearly 100,000 single star - binary encounters. They explored much of the available parameter space, and found that the current binary in fact could be formed dynamically by an exchange encounter (after subsequent tidal circularization). However, producing a product binary that remains bound to the cluster is a significant additional constraint that rules out most encounter paths to the current binary. Only $0.11 \%$ of the simulated encounters produced a binary similar to 3002 that would still be bound to the cluster. The next step, currently underway, is to determine the probability 
of a horizontal-branch single star - binary encounter given an assumed binary population and cluster mass function.

In summary, an essential point of all three cases is that we are identifying candidate products of dynamical encounters in real clusters and approaching the capability to explore numerically specific formation paths, as compared to mere qualitative conjectures. Of course, accurate injection of hydrodynamics awaits, but this too on the horizon. Perhaps the most significant challenge will soon no longer be technical, but rather proper understanding of a posteriori simulations.

\section{Tidal Circularization Rates in Solar-type Binary Stars}

Tidal effects play major roles in the close stellar encounters possible in resonant binarysingle star and binary-binary encounters, and indeed tidal rates can be the difference between a close fly-by, the formation of a close binary, or a merger. As such, tidal physics may impact significantly on the accuracy of dynamical simulations.

For two decades, open clusters have been prime laboratories for the study of tidal circularization rates. Given a coeval sample of binaries in a cluster, theory predicts that the shortest period binaries will be tidally circularized while longer period binaries will retain their primordial orbital eccentricities. The orbital period at the boundary between these two eccentricity domains, or the tidal circularization period, is set by tidal circularization rates and, in most theories, the cluster age.

The most recent and extensive study of tidal circularization periods in open clusters is that of Meibom \& Mathieu (2005). Fig. 8, taken from their paper, summarizes the

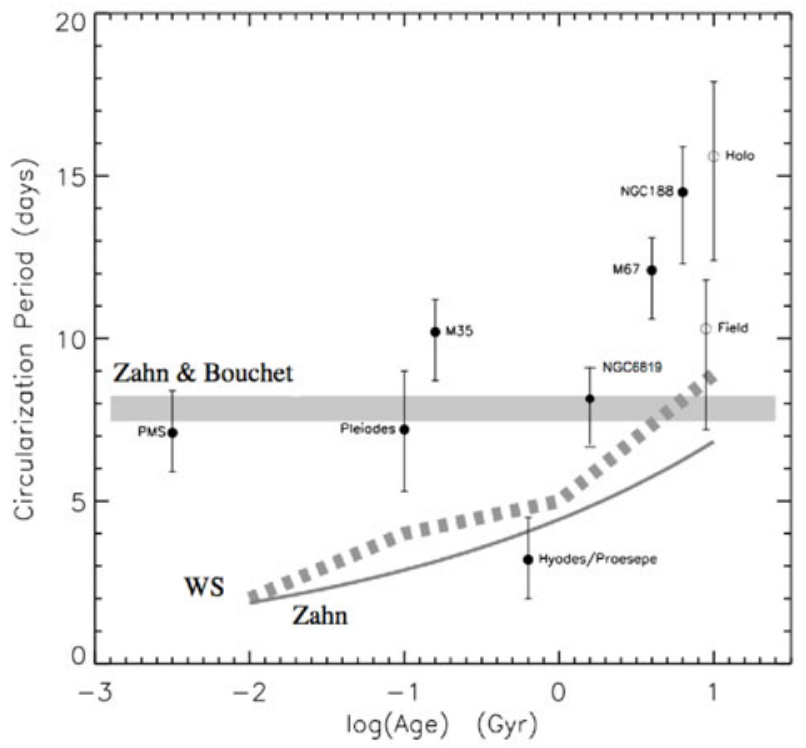

Figure 8. Distribution of tidal circularization periods with age for eight solar-type binary populations. The solid curve shows the predicted cutoff period as a function of time based on main-sequence tidal circularization using the revised equilibrium tide theory by Zahn (1989). The broad dashed band represents the predicted cutoff period for initially supersynchronous 1 $\mathrm{M}_{\odot}$ stars calculated in the framework of the dynamical tide model including resonance locking (Witte \& Savonije 2002). The horizontal gray band represents the prediction by Zahn \& Bouchet (1989), in which tidal circularization is significant only during the pre-main-sequence phase. (Taken from Meibom \& Mathieu 2005.) 
essential results. With the exception of the Hyades/Praesepe population, the distribution of tidal circularization periods with age shows an increase from the pre-main-sequence to the late main sequence. The theoretical models of main-sequence tidal circularization using either the equilibrium tide theory (Zahn 1989) or the dynamical tide theory with resonance locking (Witte \& Savonije 2002) predict longer cutoff periods with increasing population age, in agreement with the trend in the distribution of circularization periods. However, the predicted circularization periods fall significantly below the observed circularization periods. This suggests that the efficiency of the dissipation in these models is too low. Alternative, Zahn \& Bouchet (1989) suggest that all tidal circularization takes place during the pre-main-sequence phase when the stars are large with deep convective zones, and that no further circularization takes place during the main-sequence stage. This theoretical model does not predict the observed increase in tidal circularization periods with increasing cluster age.

The essential result of these studies is that actual tidal circularization rates in mainsequence solar-type stars are substantially higher than theoretical prediction. As such, the use of current theory in dynamical simulations must be done with some caution.

\section{Closing Thoughts}

For the last half-century, N-body simulations lagged behind observations of actual star clusters, the latter having the advantage of "the great analog computer in the sky". Thus attempts to compare N-body simulations with star clusters were limited by the models.

I would suggest that we are entering a period during which the simulations will catch up with, and indeed in certain areas will pull ahead of, the observations. At least for open clusters, the true numbers of stars in even the more massive clusters (at birth) are accessible to current computational capabilities. Equally importantly these numbers now can include high frequencies of primordial binaries. Normal stellar evolution is being computed in "real" time. And the next frontier of hydrodynamical interactions between stars is being crossed on numerous fronts, including mass transfer, tidal effects, and collisions.

One domain of stellar dynamics where the simulations have always provided more insight than have observations has been dynamical encounters between binaries and other single or binary stars. While we have known for a long time that open clusters have high binary frequencies, we have had very little information on the orbital properties of the binaries. As shown in this paper, that gap is being closed, at least for the hard binaries. It is worth mentioning, though, that the majority of post-encounter binaries and single stars are not identifiable as such from their specific properties. Thus observational studies of dynamical encounters in clusters will likely require statistical comparisons of binary populations as a function of age.

I would like to close with a more philosophical thought on the scientific method in our field, and specifically on the issue of a priori versus a posteriori modeling. One of the truly beautiful aspects of the Hurley et al. experiment for M67 was that their simulations made a priori testable predictions about the cluster, as demonstrated in Section 2. True, the Hurley et al. models were a posteriori in the sense that they sought to match their outcomes to an existing cluster, M67. However, the interfaces available to them for that comparison were primarily the color-magnitude diagram (especially the products of dynamical encounters such as blue stragglers) and the current mass functions. The interface with the binary populations was not accessible, simply because our work was not yet done, so they made a priori predictions about the current binary population.

That aspects of their predicted binary population may not agree with reality, based on NGC 188, is not nearly as significant as the fact that we are now in a place where N-body 
simulations can make meaningful, detailed predictions of observables. Associated with this capability is the fundamental question of how to proceed. At the time of the Hurley et al. calculations, creating an M67 was still a substantial task in terms of real time; thus very soon (even now?) such calculations will be easily doable. Given this capability, I suggest that we will want to transition from trying to match specific real clusters such as M67 a posteriori to building a priori arrays of clusters that we compare as an ensemble to observed clusters.

For N-body simulators, learning about observations of clusters is a bit like losing the innocence of youth - it can be difficult to maintain an a priori independence when one knows the answer. Arguably to ignore observations would be (computationally) inefficient in any case. Nonetheless, perhaps in these early days we will be able to continue to enjoy the pleasure of simulations like those of Hurley et al. with predictions that are both a priori and testable, mimicking the way that we teach our students that science "really works"!

\section{Acknowledgements}

I am deeply indebted to the many fine students who have done much of the work reported here, including Ella Braden, Michael DiPompeo, Christopher Dolan, Aaron Geller, Natalie Gosnell, Soeren Meibom, Meagan Morscher, Keivan Stassun, and Sylvana Yelda. I also want to acknowledge the superb staff of the WIYN Observatory who have made the acquisition of many thousand of superb stellar spectra both efficient and fun. This work was funded by National Science Foundation grant AST-0406615.

\section{References}

Albrow, M. D., Gilliland, R. L., Brown, T. M., Edmonds, P. D., Guhathakurta, P., \& Sarajedini, A. $2001, A p J, 559,1060$

Duquennoy, A. \& Mayor, M. 1991, A\&SA 248, 485

Fregeau, J. M., Cheung, P., Portegies Zwart, S. F., \& Rasio, F. A. 2004, MNRAS, 352, 1

Geller, A. M., Mathieu, R. D., Harris, H. C., \& McClure, R. D. 2008, AJ, submitted

Goranskij, V. P., Kusakin, A. V., Mironov, A. V., Moshkaljov, V. G., \& Pastukhova, E. N. 1992, $A \mathscr{E A T}, 2,201$

Gosnell, N. M., DiPompeo, M. A., Braden, E. K., Geller, A. M., \& Mathieu, R. D., 2008, BAAS, in press

Hurley, J. R., Pols, O. R., Aarseth, S. J., \& Tout, C. A. 2005, MNRAS, 363, 293

Kim, Y. C., Demarque, P., Yi, S. K., \& Alexander, D. R. 2002, ApJS, 143, 499

Kroupa, P. 1995, MNRAS, 277, 1507

Mathieu, R. D. 2000, ASP Conf. Ser., 198, 517

Mathieu, R. D., Meibom, S., \& Dolan, C. J. 2004, ApJ, 602, L121

Mathieu, R. D. \& Latham, D. W. 1986, AJ, 92, 1364

Mathieu, R. D., van den Berg, M., Torres, G., Latham, D., Verbunt, F., \& Stassun, K. 2003, AJ, 125, 246

McNamara, B. J. \& Sekiguchi, K. 1986, ApJ, 310, 613

Meibom, S. \& Mathieu, R. D. 2005, ApJ, 620, 970

Sandquist, E. L., Latham, D. W., Shetrone, M. D., \& Milone, A. A. E. 2003, AJ, 125, 810

Tinsley, B. M. \& King, I. R. 1976, AJ, 81, 835

van den Berg, M., Orosz, J., Verbunt, F., \& Stassun, K. 2001, A $\& A$, 375, 375

Witte, M. G. \& Savonije, G. J. 2002, A\&A, 386, 222

Zahn, J.-P. 1989, A\&A, 220, 112

Zahn, J.-P. \& Bouchet, L. 1989, A\&AA, 223, 112 